\title{
Image Quality of Prospectively ECG-Triggered Coronary CT Angiography in Heart Transplant Recipients
}

\author{
Andrea Bartykowszki ${ }^{1}$ \\ Márton Kolossváry ${ }^{1}$ \\ Ádám Levente Jermendy ${ }^{1}$ \\ Júlia Karády ${ }^{1}$ \\ Bálint Szilveszter ${ }^{1}$ \\ Mihály Károlyi ${ }^{1}$ \\ Orsolya Balogh2 \\ Balázs Sax ${ }^{1}$ \\ Béla Merkely ${ }^{1}$ \\ Pál Maurovich-Horvat ${ }^{1}$
}

Keywords: coronary CT angiography, heart transplantation, image quality

doi.org/10.2214/AJR.17.18546

B. Merkely and P. Maurovich-Horvat contributed equally to this work.

Received May 27, 2017; accepted after revision August 16, 2017.

Based on a presentation at the European Congress of Radiology 2017 annual meeting, Vienna, Austria.

Supported by grant NVKP-16-1-2016-0017 from the National Research, Development, and Innovation Office of Hungary.

${ }^{1}$ MTA-SE Cardiovascular Imaging Research Group, Heart and Vascular Center, Semmelweis University, 68 Városmajor St, Budapest H-1122, Hungary. Address correspondence to P. Maurovich-Horvat (p.maurovich.horvat@mail.harvard.edu).

${ }^{2}$ Department of Cardiology, Gottsegen György National Cardiology Institute, Budapest, Hungary.

AJR2018; 210:314-319

$0361-803 X / 18 / 2102-314$

(C) American Roentgen Ray Society

OBJECTIVE. Cardiac allograft vasculopathy (CAV) is among the top causes of death 1 year after heart transplantation (HTx). Coronary CT angiography (CTA) is a potential alternative to invasive imaging in the diagnosis of CAV. However, the higher heart rate (HR) of HTx recipients prompts the use of retrospective ECG-gating, which is associated with higher radiation dose, a major concern in this patient population. Therefore, we sought to evaluate the feasibility and image quality of low-radiation-dose prospectively ECG-triggered coronary CTA in HTx recipients.

MATERIALS AND METHODS. In total, 1270 coronary segments were evaluated in 50 HTx recipients and 50 matched control subjects who did not undergo HTx. The control subjects were selected from our clinical database and were matched for age, sex, body mass index, HR, and coronary dominance. Scans were performed using 256-MDCT with prospective ECG-triggering. The degree of motion artifacts was evaluated on a per-segment basis on a 4-point Likert-type scale.

RESULTS. The median HR was 74.0 beats/min (interquartile range [IQR], 67.8-79.3 beats/min) in the HTx group and 73.0 beats $/ \mathrm{min}$ (IQR, $68.5-80.0$ beats $/ \mathrm{min}$ ) in the matched control group $(p=0.58)$. In the HTx group, more segments had diagnostic image quality compared with the control group (624/662 [94.3\%] vs 504/608 [82.9\%]; $p<0.001)$. The mean effective radiation dose was low in both groups (3.7 $\mathrm{mSv}$ [IQR, 2.4-4.3 mSv] in the HTx group vs $4.3 \mathrm{mSv}$ [IQR, $2.6-4.3 \mathrm{mSv}$ ] in the control group; $p=0.24)$.

CONCLUSION. Prospectively ECG-triggered coronary CTA examinations of HTx recipients yielded diagnostic image quality with low radiation dose. Coronary CTA is a promising noninvasive alternative to routine catheterization during follow-up of HTx recipients to diagnose CAV.

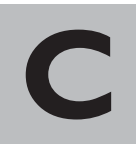

ardiac allograft vasculopathy $(\mathrm{CAV})$ is the leading cause of death during the first year after heart transplantation (HTx). The overall frequency of CAV at 1,5 , and 10 years after transplantation is $8 \%, 30 \%$, and $50 \%$, respectively [1]. CAV is characterized by diffuse concentric intimal hyperplasia [2]. Because of the denervated transplanted hearts, patients do not experience symptoms related to ischemia; therefore, early diagnosis of $\mathrm{CAV}$ is challenging. International guidelines recommend annual or biannual invasive coronary angiography for the assessment of coronary status. However, invasive coronary angiography has limited diagnostic accuracy to detect CAV because of the diffuse and concentric manifestation of the disease. Furthermore, invasive coronary angiography does not provide information regarding the coro- nary wall; therefore, intravascular ultrasound or optical coherence tomography is suggested as a complementary imaging test [3]. The combination of invasive coronary angiography with intravascular imaging techniques increases sensitivity, but their routine use increases costs and rates of procedural complications; therefore, it is considered optional for CAV assessment [4]. In addition, the International Society for Heart and Lung Transplantation consensus statement does not recommend the routine use of intravascular ultrasound for CAV assessment [3].

Coronary CT angiography (CTA) allows noninvasive visualization of the coronary artery wall and lumen with a high diagnostic accuracy [5,6]. It can detect 1.5-2 times more coronary segments with coronary atherosclerotic plaques than does invasive coronary angiography [7]. Notably, the absence of para- 


\section{Quality of Coronary CTA Images of HTx Recipients}

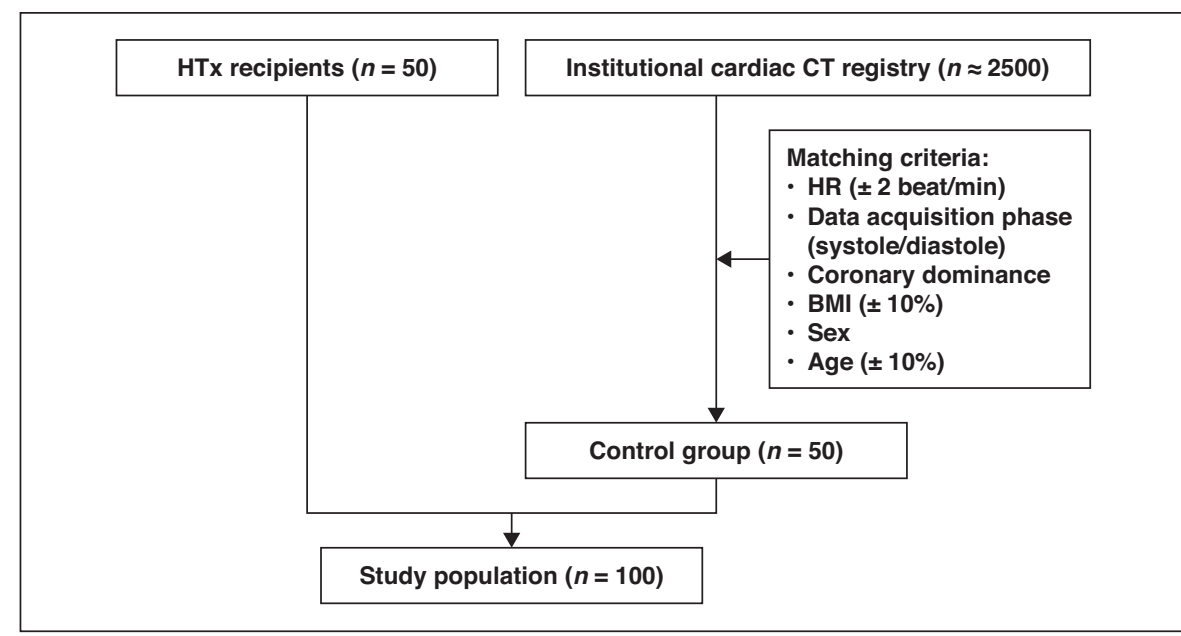

Fig. 1-Flowchart of study population selection. BMI = body mass index (weight in kilograms divided by the square of height in meters), $\mathrm{HR}=$ heart rate, $\mathrm{HTx}=$ heart transplantation. sympathetic and sympathetic innervation of the transplanted hearts results in higher resting heart rates (HRs), which may compromise the diagnostic performance of coronary CTA. Moreover, because of their higher HRs, retrospective ECG-gating has been used for HTx recipients, which results in higher radiation dose. These concerns precluded the widespread use of coronary CTA in HTx recipients [8]. Prospectively ECG-triggered coronary CTA would be desirable because of its low radiation dose, but it requires a low HR (generally $<65$ beats/min). The HTx recipients have higher but steady HR with minimal HR variability because of the lack of autonomic innervation. The steady HR of HTx recipients might provide a unique opportunity to scan these patients with low radiation dose and achieve good image quality. There-

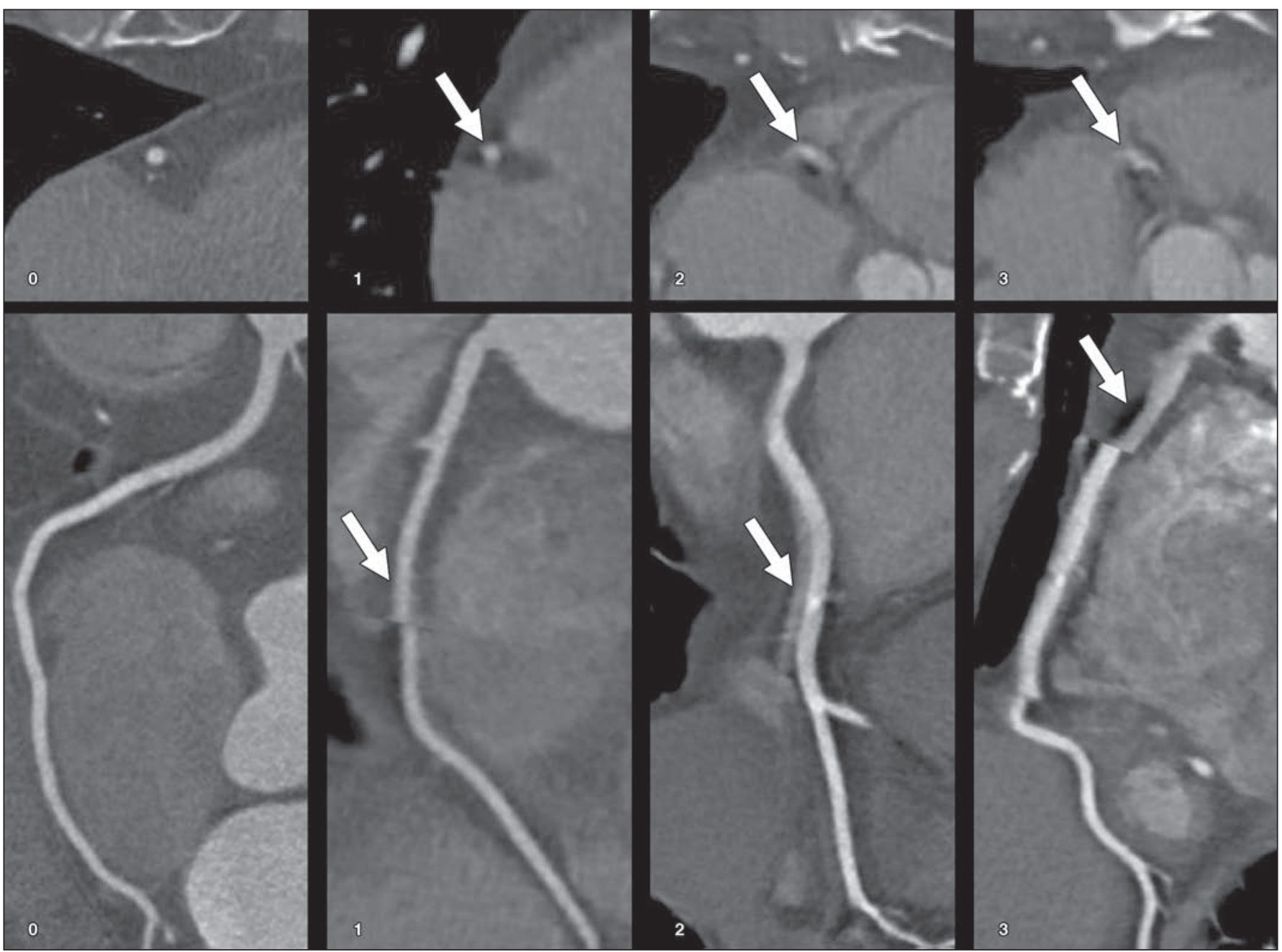

Fig. 2-Examples of 4-point Likert scale of motion artifacts in heart transplant recipients: 0, excellent image quality with no artifacts (62-year-old man); 1 , good image quality with minor artifacts (60-year-old woman); 2 , moderate image quality, acceptable for routine clinical diagnosis (44-year-old woman); 3 , not evaluable, with severe artifacts impairing accurate evaluation (60-year-old man). Upper panels show cross-sectional CT angiography images of right coronary arteries with different motion artifact severities. Lower panels show same vessels in curved multiplanar reconstructions. Arrows indicate motion artifacts. 


\section{Bartykowszki et al.}

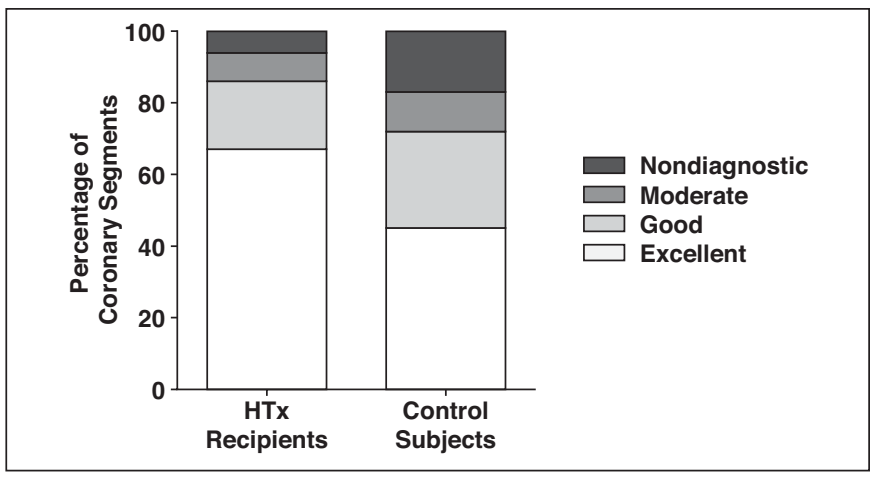

fore, our aim was to assess the image quality of low-dose prospectively ECG-triggered coronary CTA in HTx recipients.

\section{Materials and Methods}

In a retrospective matched case-control cohort study, we evaluated the image quality of coronary CTA performed of HTx recipients. The institutional review board of Semmelweis University approved the study (approval number SE-TUKEB 173/2016), and because of the retrospective study design, informed consent was waived. The study was conducted in compliance with the Helsinki declaration.

During a 4-year period, 97 coronary CTAs were performed of $57 \mathrm{HTx}$ recipients to rule out CAV. If a patient underwent more than one scan, the scan obtained with the highest HR was selected. Scans with breathing artifacts $(n=3)$, contrast agent extravasation $(n=1)$, and high image noise or insufficient contrast opacification $(n=3)$ were excluded from the study. In total, 50 HTx recipients (HTx group) were included in the study. The image quality of the scans of the HTx recipients was compared with that of scans of a control group of patients who did not undergo HTx. The control group was selected from our institutional cardiac CT registry. We selected the control group according to matching criteria that may influence image quality: age, sex, body mass index (weight in kilograms divided by the square of height in meters), HR, data acquisition phase (systole or diastole), and coronary dominance (Fig. 1). For the HR, a maximum difference of \pm 2 beats/min was allowed; for body mass index and age, a maximum difference of $\pm 10 \%$ was allowed. In addition, we matched every pair for coronary dominance. Codominant coronary system was regarded as left dominant.

All patients underwent imaging with a 256MDCT scanner (Brilliance iCT 256, Philips Healthcare). Tube voltage was $100-120 \mathrm{kV}$, and the tube current was set to $100-300 \mathrm{~mA}$ depending on the body mass index of the patients. Collimation was $2 \times 128 \times 0.625 \mathrm{~mm}$, with a gantry rotation time of $270 \mathrm{~ms}$. Both the HTx recipients and
Fig. 3-Proportions of coronary segments with nondiagnostic, moderate, good, and excellent image quality in heart transplantation ( $\mathrm{HTX}$ ) recipients and control subjects. the control group were scanned with a prospectively ECG-triggered acquisition mode. When the HR was over 80 beats/min, systolic triggering was used at $40 \%$ of the cardiac cycle with $3 \%$ padding (37-43\% of the R-R interval); in all other cases, diastolic triggering was used at $78 \%$ of the cardiac cycle with $3 \%$ padding ( $75-81 \%$ of the R-R interval) [9]. We used a four-phase contrast injection protocol with iodinated contrast agent (iomeprol, $400 \mathrm{mg} \mathrm{I} / \mathrm{mL}$; Iomeron 400, Bracco), with a flow rate of $4.5-5.5 \mathrm{~mL} / \mathrm{s}$ with an extra saline bolus preceding the contrast bolus [10]. A bolustracking technique was used with an ROI in the left atrium. For HR control, we used 7.5-15 mg ivabradine (Procorolan, $5 \mathrm{mg}$, Les Laboratoires Servier) administered 3 hours before the scan in $90 \%$ of HTx recipients and $50-100 \mathrm{mg}$ oral metoprolol and 5-20 mg IV metoprolol (Betaloc, 1 $\mathrm{mg} / \mathrm{mL}$, AstraZeneca; 5-mg ampoule) in 58\% and $48 \%$ of control subjects, respectively. All patients received $0.8 \mathrm{mg}$ of sublingual nitroglycerin $(\mathrm{Ni}-$ tromint, $8 \mathrm{mg} / \mathrm{g}$, EGIS) a maximum of 1 minute

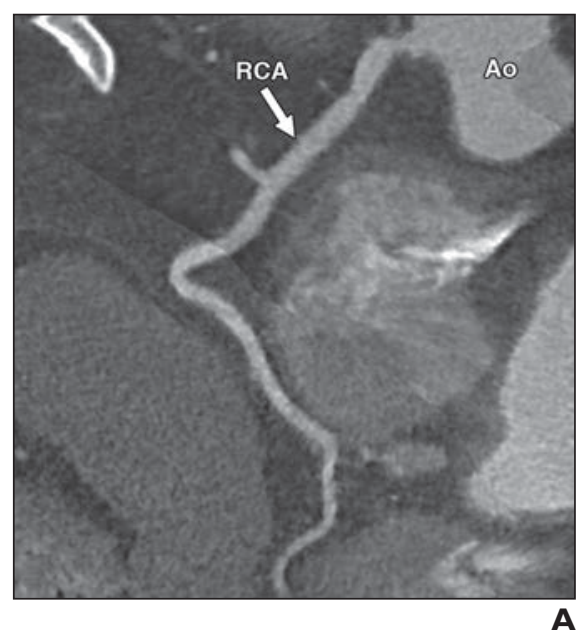

before the image acquisition. Images were reconstructed with $0.8-\mathrm{mm}$ slice thickness and $0.4-\mathrm{mm}$ increment using a hybrid iterative reconstruction (iDOSE 4 , Philips Healthcare) technique.

Reconstructed images were evaluated by two readers (with 5 and 3 years of experience in coronary CTA) using the 18 -segment model of the Society of Cardiovascular CT [11]. Coronary segments with a diameter greater than $1.5 \mathrm{~mm}$ were assessed. We used axial images, multiplanar reformations, and maximum intensity projections to evaluate the image quality. Motion artifacts were described in every coronary segment using a 4-point Likert scale: 0 , excellent image quality with no artifacts; 1 , good image quality with minor artifacts; 2 , moderate image quality, acceptable for routine clinical diagnosis; and 3, not evaluable, with severe artifacts impairing accurate evaluation [12, 13] (Fig. 2). To quantify the total amount of motion artifacts on a per-patient level, we defined the segment motion score, which describes how many segments had motion artifact, and the segment Likert score, which is the sum of the motion severity Likert score of the patient. Because the number of coronary segments affects the total obtainable score, we normalized the scores by dividing them by the number of segments present, which resulted in the segment motion score index and segment Likert score index. To describe how many nondiagnostic segments were present, we defined the segment nondiagnostic score and also divided it by the number of the evaluated segments, which yielded the segment nondiagnostic score index. Furthermore, to assess the effect of systolic versus diastolic triggering, we conducted a subgroup analysis among both HTx recipients and control subjects.

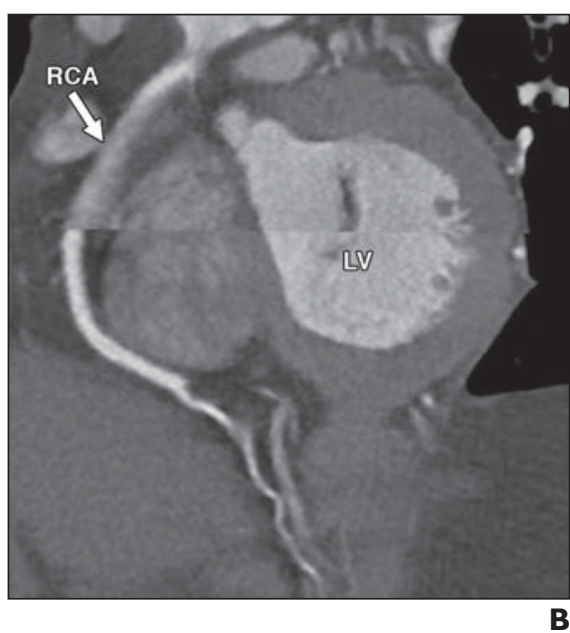

Fig. 4-Coronary CT angiograms of heart transplant recipient and age- and sex-matched control subject. A, 48-year-old male heart transplant recipient with heart rate of 75 beats $/ \mathrm{min}$. No motion artifact is visible in right coronary artery (RCA; arrow) on curved multiplanar reconstruction. $A_{0}=$ aorta.

B, 48-year-old man with heart rate of 75 beats/min who did not receive heart transplant. Motion artifact (arrow) is visible in proximal segment of RCA on curved multiplanar reconstruction. $L V=$ left ventricle. 


\section{Quality of Coronary CTA Images of HTx Recipients}

The Shapiro-Wilk test was used to assess normality. Because all continuous variables showed nonnormal distribution, continuous variables are expressed as median and interquartile range (IQR). Categoric variables are expressed as numbers and percentages. The Mann-Whitney $U$ test was used to compare continuous data of the HTx and non-HTx groups. Categoric data were compared using the chi-square test. Intrareader and interreader reproducibility was assessed on the basis of 20 randomly selected individuals' images using Cohen kappa, interpreted as follows: $1.00-0.81$, excellent; 0.80 0.61 , good; $0.60-0.41$, moderate; $0.40-0.21$, fair; and $0.20-0.00$, poor $[14,15]$. All statistical calculations were done using SPSS software (version 23 , IBM). A $p<0.05$ was considered significant.

\section{Results}

In total, $50 \mathrm{HTx}$ recipients were included in our study. Every HTx recipient had a matched control subject who did not undergo HTx; therefore, 100 subjects in total were evaluated. In the HTx group (11 women [22\%]; 4.3 years after transplantation), the median age was 57.9 years (IQR, 46.7-59.9 years) and the median HR was 74.0 beats/ min (IQR, 67.8-79.3 beats/min), compared with 73.0 beats/min (IQR, 68.5-80.0 beats/ $\min )$ in the matched control group $(p=0.58)$. We found no significant difference between the HTx and control groups regarding anthropometric data and scan characteristics (Table 1). The effective radiation dose was relatively low in both groups $(3.7 \mathrm{mSv}$ [IQR, 2.4$4.3 \mathrm{mSv}$ ] in the HTx group vs $4.3 \mathrm{mSv}$ [IQR, $2.6-4.3 \mathrm{mSv}]$ in the control group; $p=0.24$ ).

In total, 1270 coronary segments were evaluated, 662 segments in the HTx group and 608 segments in the control group. The distribution of motion scores between the two groups is shown in Figure 3. In the HTx group, more segments (624; 94.3\%) had diagnostic image quality compared with the control group $(504 ; 82.9 \%)(p<0.001)$ (Fig. 4). In the HTx group, more segments had excellent image quality than in the control group (442 [66.7\%] vs 271 [4.5\%]; $p<$ 0.001). Furthermore, in the HTx group the number of nondiagnostic segments was approximately one-third of that of the control group (38 [5.8\%] vs 104 [17.1\%]; $p<0.001$ ).

We a found a significant difference between the two groups regarding the segment Likert score, the segment motion score, and the segment nondiagnostic score indexes. The segment Likert score index of the HTx group was approximately half that of the control group (0.4 [IQR, 0.1-0.9] vs 0.9

TABLE I: Clinical Characteristics of Study Subjects

\begin{tabular}{l|c|c|c}
\hline \multicolumn{1}{c|}{ Parameters } & $\begin{array}{c}\text { Heart Transplant } \\
\text { Recipients }(n=50)\end{array}$ & $\begin{array}{c}\text { Control Subjects } \\
(n=50)\end{array}$ & $p$ \\
\hline Age (y) & $57.9(46.7-59.9)$ & $58.6(48.5-62.1)$ & 0.32 \\
Body mass index ${ }^{\text {a }}$ & $25.0(22.6-26.5)$ & $25.0(23.1-28.4)$ & 0.45 \\
Diastolic triggering, no. (\%) of patients & $31(62.0)$ & $31(62.0)$ & 1.00 \\
Tube voltage (kV) & $120.0(100.0-120.0)$ & $120.0(100.0-120.0)$ & 0.63 \\
Tube current (mA) & $300.0(250.0-300.0)$ & $300.0(300.0-300.0)$ & 0.14 \\
Effective dose (mSv) & $3.7(2.4-4.3)$ & $4.3(2.6-4.3)$ & 0.24 \\
Contrast agent (mL) & $90.0(90.0-95.0)$ & $90.0(90.0-95.0)$ & 0.62 \\
Heart rate (beats/min) & $74.0(67.8-79.3)$ & $73.0(68.5-80.0)$ & 0.58 \\
Coronary dominance, no. (\%) of patients & & & 0.91 \\
Right dominant & $39(78.0)$ & $39(78.0)$ & \\
Left dominant & $11(22.0)$ & $11(22.0)$ & \\
\hline
\end{tabular}

Note-Except where noted otherwise, data are median (interquartile range).

aWeight in kilograms divided by the square of height in meters.

[IQR, 0.3-1.6]; $p=0.003)$. Similarly, a nearly twofold difference was found between the HTx and control groups regarding segment motion score index $(0.3$ [IQR, $0.1-0.5]$ vs 0.6 [IQR, 0.2-0.9]; $p=0.001)$. The segment nondiagnostic score index was lower in the HTx group than in the control group (0.0 [IQR, $0.0-0.1$ ] vs 0.1 [IQR, $0.0-0.3$ ]; $p=0.004$ ).

The image quality was better in HTx recipients than in control subjects in the subgroup with systolic triggering. This was reflected by the difference in the segment Likert score index, which was significantly lower in the HTx group than in the control group (0.5 [IQR, 0.4-0.7] vs 0.8 [IQR, 0.8-0.9]; $p<0.001$ ). Furthermore, among scans with systolic triggering, we found significantly fewer motion artifacts and more diagnostic segments in the HTx group; their segment motion score index was almost half that of the control group (0.8 [IQR, 0.5-1.1] vs 1.5 [IQR, 1.3-2.1]; $p<$ 0.001 ), whereas their segment nondiagnostic score index was almost one-fourth that of the control group (0.07 [IQR, 0.0-0.1] vs 0.3 [IQR, 0.1-0.5]; $p=0.001)$.

Among diastolic images, significantly better image quality was observed in the HTx scans compared with the non-HTx scans; the segment Likert score index was significantly lower in the HTx group compared with the non-HTx group (0.1 [IQR, 0.0-0.3] vs 0.4 [IQR, 0.1-0.6]; $p=0.03$ ). However, among scans with diastolic triggering, the degree of motion and the number of nondiagnostic segments did not differ significantly between the HTx recipients and control subjects (segment motion score index, 0.1 [IQR, 0.0-0.4] vs 0.5 [IQR, 0.1-1.1], $p=0.05$; segment non- diagnostic score index, 0.0 [IQR, $0.0-0.1]$ vs 0.0 [IQR, 0.0-0.1], $p=0.20$ ). The median HR with systolic triggering was 78.0 beats/min for HTx recipients compared with 80.0 beats/ min for control subjects $(p=0.86$ ); the median HR with diastolic triggering was 69.0 beats/min in HTx recipients compared with 70.0 beats/min in control subjects $(p=0.96)$.

Intrareader and interreader agreement for image quality scores was good $(\kappa=0.72$ and $\kappa=0.62$, respectively). Dichotomization of image quality scores to excellent and nonexcellent image quality scores resulted in excellent intrareader $(\kappa=0.83)$ and good interreader $(\kappa=0.69)$ reproducibility. Dichotomization to diagnostic and nondiagnostic image quality scores also showed excellent intrareader $(\kappa=0.82)$ and good interreader $(\kappa=0.73)$ reproducibility.

\section{Discussion}

In this retrospective matched case-control study, we found that scans of HTx recipients had better coronary CTA image quality than did scans of a matched control group with similar HRs. Despite the relatively high HR of HTx recipients, the number of nondiagnostic segments was low (5.8\%), suggesting that coronary CTA with prospective ECGtriggering is a robust diagnostic tool with low radiation dose in this patient population. The subgroup analysis comparing the image quality of the two groups among scans with systolic and diastolic triggering showed similar results. The HTx recipients had better overall image quality compared with the control subjects both with systolic and diastolic triggering. However, the segment mo- 


\section{Bartykowszki et al.}

tion score index did not show any difference between the two groups among the scans triggered in diastole, which is most probably due to the lower HR of patients undergoing coronary CTA with diastolic triggering.

$\mathrm{CAV}$ is among the top three causes of death 1 year after HTx. Invasive coronary angiography is considered the reference standard method to diagnose CAV. However, it has been found that diagnosis based on a single invasive coronary angiography is challenging because of the concentric intimal hyperplasia; furthermore, the interobserver variation is high [16]. Numerous studies investigated the diagnostic performance of coronary CTA to identify CAV [17-23]. von Ziegler et al. [19] studied 26 consecutive patients with a mean ( \pm SD) HR of $86 \pm 13$ beats/min using $64-M D C T$. They found that $81.4 \%$ of the segments had diagnostic image quality. According to their results, coronary CTA has high negative predictive value $(99.7 \%)$, and they concluded that coronary CTA is a reliable diagnostic tool to rule out CAV in HTx recipients [19]. Similar results were shown by Mittal et al. [17], who evaluated 130 HTx recipients (mean HR, $82.7 \pm 4$ beats $/ \mathrm{min}$ ) with 64-MDCT. Most of the evaluated segments (98\%) had diagnostic image quality. They concluded that coronary CTA has high sensitivity, specificity, and excellent negative predictive value for the diagnosis of CAV in HTx recipients in comparison with invasive angiography [17]. A meta-analysis published by Wever-Pinzon et al. [24] showed that the combined overall weighted mean sensitivity, specificity, positive predictive value, and negative predictive value of CT for detection of CAV were $97 \%, 81 \%, 78 \%$, and $97 \%$, respectively. Importantly, these studies used retrospective ECG-gating, because of the higher resting HR of HTx recipients, which resulted in higher radiation dose (10.2-17.5 mSv) $[17,22,23]$. The reduction of radiation dose is of utmost importance for HTx recipients who undergo repeated scans and take immunosuppressant therapy, which substantially increases cancer risk.

We found that HTx recipients can be scanned with a prospective ECG-triggering scan mode with a low radiation dose (mean effective radiation dose, $3.7 \mathrm{mSv}$ ). Furthermore, we found that scans of HTx recipients with median HR of 74.0 beats/min have significantly better image quality than scans of control subjects with a similar HR (73.0 beats/min). In addition, the number of segments with excellent image quality was high- er for HTx recipients than for control subjects. In accordance with these results, the ratio of nondiagnostic segments was lower among HTx recipients.

Our observations might be explained by the loss of autonomous neural control. The surgical denervation after heart transplantation causes chronotropic incompetence, which results in elevated resting $\mathrm{HR}$ and nearly absent HR variability [25-27]. According to Stolzmann et al. [28] and Brodoefel et al. [29], HR variability has a significant effect on the image quality in prospectively triggered coronary CTA. Therefore, the lack of autonomous neural control and the consequent regular and steady HR seems to be optimal for prospectively ECGtriggered coronary CTA.

Despite the excellent diagnostic accuracy and low radiation dose of modern CT scanners, the routine use of coronary CTA for follow-up of HTx recipients has not become widely accepted in daily practice. The guidelines of the International Society for Heart and Lung Transplantation [3] and the recommendations of the European Association of Cardiovascular Imaging and Cardiovascular Imaging Department of the Brazilian Society of Cardiology [8] raise concerns regarding the higher HR (higher than the optimal 65 beats/min) of HTx recipients and the excess ionizing radiation. However, contemporary scanner technology allows coronary imaging at ever decreasing radiation doses, and, with modern scanners, the radiation dose generally does not exceed that associated with invasive coronary angiography $[30,31]$. Therefore, we believe that, in experienced centers with contemporary CT scanners, coronary CTA is a promising alternative to invasive coronary angiography for follow-up of HTx recipients.

This study has some limitations that we have to acknowledge. First, coronary segments with a diameter smaller than $1.5 \mathrm{~mm}$ were excluded from the study because of the limited accuracy of coronary CTA in distal coronary segments and small-caliber side braches. Nevertheless, the evaluation of small coronary segments remains a challenge even with invasive techniques.

Second, because of the specific postoperative appearance of the extracardiac structures (e.g., sternal sutures and great vessel anastomoses) of HTx recipients, the readers could not be blinded to the compared groups (HTx recipients vs control subjects), which might represent a potential bias that affects quality rating. Furthermore, we acknowledge that this study was a single-center single-vendor study using a 256-MDCT scanner, which might limit the generalizability of our findings.

In conclusion, coronary CTA of HTx recipients had significantly better image quality compared with a control group with similar HRs. This finding suggests that invasive coronary angiography could be replaced by coronary CTA in experienced centers to diagnose CAV. In addition, a higher optimal HR threshold might be recommended for coronary CTA among HTx recipients because of the lack of autonomous innervation of the heart and diminished HR variability. With the use of coronary CTA in the clinical routine, the burden of invasive investigations could be reduced in this vulnerable patient population.

\section{References}

1. Lund LH, Edwards LB, Kucheryavaya AY, et al. The registry of the International Society for Heart and Lung Transplantation: thirty-first official adult heart transplant report-2014; focus theme: retransplantation. J Heart Lung Transplant 2014; 33:996-1008

2. Park KH, Kwon TG, Matsuzawa Y, et al. Association between the vasa vasorum and the atherosclerotic changes in cardiac allograft vasculopathy: volumetric analysis. Eur Heart J Cardiovasc Imaging 2016; 17:272-279

3. Mehra MR, Crespo-Leiro MG, Dipchand A, et al. International Society for Heart and Lung Transplantation working formulation of a standardized nomenclature for cardiac allograft vasculopathy: 2010. J Heart Lung Transplant 2010; 29:717-727

4. Costanzo MR, Dipchand A, Starling R, et al. The International Society of Heart and Lung Transplantation Guidelines for the care of heart transplant recipients. J Heart Lung Transplant 2010; 29:914-956

5. Achenbach S, Raggi P. Imaging of coronary atherosclerosis by computed tomography. Eur Heart J 2010; 31:1442-1448

6. Maurovich-Horvat P, Ferencik M, Voros S, Merkely B, Hoffmann U. Comprehensive plaque assessment by coronary CT angiography. Nat Rev Cardiol 2014; 11:390-402

7. Kolossváry M, Szilveszter B, Edes IF, et al. Comparison of quantity of coronary atherosclerotic plaques detected by computed tomography versus angiography. Am J Cardiol 2016; 117:1863-1867

8. Badano LP, Miglioranza MH, Edvardsen T, et al. European Association of Cardiovascular Imaging/Cardiovascular Imaging Department of the Brazilian Society of Cardiology recommendations for the use of cardiac imaging to assess and follow patients after heart transplantation. Eur Heart J Cardiovasc Imaging 2015; 16:919-948 


\section{Quality of Coronary CTA Images of HTx Recipients}

9. Lee AM, Beaudoin J, Engel LC, et al. Assessment of image quality and radiation dose of prospectively ECG-triggered adaptive dual-source coronary computed tomography angiography (cCTA) with arrhythmia rejection algorithm in systole versus diastole: a retrospective cohort study. Int $J$ Cardiovasc Imaging 2013; 29:1361-1370

10. Karády J, Panajotu A, Kolossváry M, et al. The effect of four-phasic versus three-phasic contrast media injection protocols on extravasation rate in coronary CT angiography: a randomized controlled trial. Eur Radiol 2017; 27:4538-4543

11. Leipsic J, Abbara S, Achenbach S, et al. SCCT guidelines for the interpretation and reporting of coronary CT angiography: a report of the Society of Cardiovascular Computed Tomography Guidelines Committee. J Cardiovasc Comput Tomogr 2014; 8:342-358

12. Leschka S, Alkadhi H, Plass A, et al. Accuracy of MSCT coronary angiography with 64-slice technology: first experience. Eur Heart $J$ 2005; 26:1482-1487

13. Shuman WP, Branch KR, May JM, et al. Prospective versus retrospective ECG gating for 64-detector CT of the coronary arteries: comparison of image quality and patient radiation dose. Radiology 2008; 248:431-437

14. Altman DG. Practical statistics for medical research. London, UK: Chapman and Hall, 1991

15. Landis JR, Koch GG. The measurement of observer agreement for categorical data. Biometrics 1977; 33:159-174

16. Wellnhofer E, Stypmann J, Bara CL, et al. Angiographic assessment of cardiac allograft vasculopathy: results of a Consensus Conference of the Task Force for Thoracic Organ Transplantation of the German Cardiac Society. Transpl Int 2010; 23:1094-1104

17. Mittal TK, Panicker MG, Mitchell AG, Banner NR. Cardiac allograft vasculopathy after heart transplantation: electrocardiographically gated cardiac CT angiography for assessment. Radiology 2013; 268:374-381

18. Gregory SA, Ferencik M, Achenbach S, et al. Comparison of sixty-four-slice multidetector computed tomographic coronary angiography to coronary angiography with intravascular ultrasound for the detection of transplant vasculopathy. Am J Cardiol 2006; 98:877-884

19. von Ziegler F, Leber AW, Becker A, et al. Detection of significant coronary artery stenosis with 64-slice computed tomography in heart transplant recipients: a comparative study with conventional coronary angiography. Int J Cardiovasc Imaging 2009; 25:91-100

20. Nunoda S, Machida H, Sekikawa A, et al. Evaluation of cardiac allograft vasculopathy by multidetector computed tomography and whole-heart magnetic resonance coronary angiography. Circ J 2010; 74:946-953

21. Bastarrika G, De Cecco CN, Arraiza M, et al. Dual-source CT for visualization of the coronary arteries in heart transplant patients with high heart rates. AJR 2008; 191:448-454

22. Schepis T, Achenbach S, Weyand M, et al. Comparison of dual source computed tomography versus intravascular ultrasound for evaluation of coronary arteries at least one year after cardiac transplantation. Am J Cardiol 2009; 104:13511356

23. Kępka C, Sobieszczańsk-Małek M, Pregowski J, et al. Usefulness of dual-source computed tomogra- phy for the evaluation of coronary arteries in heart transplant recipients. Kardiol Pol 2012; 70:1111-1119

24. Wever-Pinzon O, Romero J, Kelesidis I, et al. Coronary computed tomography angiography for the detection of cardiac allograft vasculopathy: a meta-analysis of prospective trials. $J$ Am Coll Cardiol 2014; 63:1992-2004

25. Sands KE, Appel ML, Lilly LS, Schoen FJ, Judge GH Jr, Cohen RJ. Power spectrum analysis of heart rate variability in human cardiac transplant recipients. Circulation 1989; 79:76-82

26. Cornelissen VA, Vanhaecke J, Aubert AE, Fagard RH. Heart rate variability after heart transplantation: a 10-year longitudinal follow-up study. J Cardiol 2012; 59:220-224

27. Willman VL, Cooper T, Cian LG, Rollins Hanlon C. Neural responses following autotransplantation of the canine heart. Circulation 1963; 27:713-716

28. Stolzmann P, Leschka S, Scheffel H, et al. Dualsource CT in step-and-shoot mode: noninvasive coronary angiography with low radiation dose. Radiology 2008; 249:71-80

29. Brodoefel H, Burgstahler C, Tsiflikas I, et al. Dual-source CT: effect of heart rate, heart rate variability, and calcification on image quality and diagnostic accuracy. Radiology 2008; 247:346-355

30. Hong YJ, Kim SJ, Lee SM, et al. Low-dose coronary computed tomography angiography using prospective ECG-triggering compared to invasive coronary angiography. Int $J$ Cardiovasc Imaging $2011 ; 27: 425-431$

31. Herzog BA, Wyss CA, Husmann L, et al. First head-to-head comparison of effective radiation dose from low-dose 64-slice CT with prospective ECG-triggering versus invasive coronary angiography. Heart 2009; 95:1656-1661 